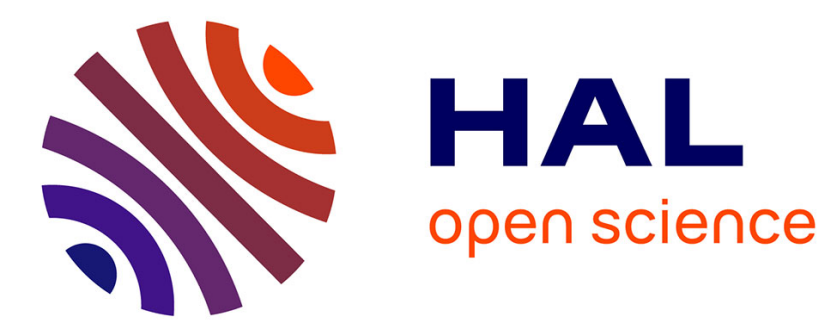

\title{
Wrinkles, folds, and plasticity in granular rafts
}

\author{
Etienne Jambon-Puillet, Christophe Josserand, Suzie Protiere
}

\section{To cite this version:}

Etienne Jambon-Puillet, Christophe Josserand, Suzie Protiere. Wrinkles, folds, and plasticity in granular rafts. Physical Review Materials, 2017, 1 (4), 10.1103/PhysRevMaterials.1.042601 . hal02172351

\section{HAL Id: hal-02172351 \\ https://hal.science/hal-02172351}

Submitted on 3 Jul 2019

HAL is a multi-disciplinary open access archive for the deposit and dissemination of scientific research documents, whether they are published or not. The documents may come from teaching and research institutions in France or abroad, or from public or private research centers.
L'archive ouverte pluridisciplinaire HAL, est destinée au dépôt et à la diffusion de documents scientifiques de niveau recherche, publiés ou non, émanant des établissements d'enseignement et de recherche français ou étrangers, des laboratoires publics ou privés. 
PHYSICAL REVIEW MATERIALS 1, 042601(R) (2017)

Wrinkles, folds, and plasticity in granular rafts

\author{
Etienne Jambon-Puillet, Christophe Josserand, and Suzie Protière \\ Sorbonne Universités, UPMC Université Paris 06, CNRS, UMR 7190, Institut Jean Le Rond d'Alembert, F-75005 Paris, France
}

(Received 19 April 2017; published 11 September 2017)

\begin{abstract}
We investigate the mechanical response of a compressed monolayer of large and dense particles at a liquid-fluid interface: a granular raft. Upon compression, rafts first wrinkle; then, as the confinement increases, the deformation localizes in a unique fold. This characteristic buckling pattern is usually associated with floating elastic sheets, and as a result, particle laden interfaces are often modeled as such. Here, we push this analogy to its limits by comparing quantitative measurements of the raft morphology to a theoretical continuous elastic model of the interface. We show that, although powerful to describe the wrinkle wavelength, the wrinkle-to-fold transition, and the fold shape, this elastic description does not capture the finer details of the experiment. We describe an unpredicted secondary wavelength, a compression discrepancy with the model, and a hysteretic behavior during compression cycles, all of which are a signature of the intrinsic discrete and frictional nature of granular rafts. It suggests also that these composite materials exhibit both plastic transition and jamming dynamics.
\end{abstract}

DOI: 10.1103/PhysRevMaterials.1.042601

Simple compression tests are commonly conducted on a material to probe its mechanical properties. When performed on an elastic film resting on a foundation, wrinkling patterns and then a localization into a single fold is observed [1-6]. This wrinkle-to-fold transition has explained in recent years the formation of patterns in various systems, such as the wrinkling of the skin, multilayer folds observed in geological layers, or the cortical folding in the fetal brain [7-9].

However, many practical situations in biology and industry involve complex membranes formed of discrete objects. Such composite interfaces can be as diverse as pulmonary surfactant monolayers, which are compressed and expanded upon exhalation, thus preventing lung collapse [10], biofilms on water that form wrinkles when confined [11], or ultrathin layers of nanoparticles placed at an air-water interface [12,13]. In addition, many fundamental studies have focused on interfaces coated with proteins, soaps, or particles since they stabilize or rigidify emulsion [14]. In this context, densely packed monolayers present some characteristics of elastic sheets: They can sustain shear and buckle out of plane to form wrinkles $[15,16]$. Different elastic moduli can also be measured by using a Langmuir-Blodgett trough, by manipulating particlecoated droplets, or by creating surface waves [15,17-21]. The possibility of particle rearrangement and jamming [22-25], together with the presence of chain forces between grains $[26,27]$, coupled with a liquid interface, suggest also that these discrete frictional sheets represent an original material more complicated than a pure elastic membrane [17,20,21,28,29]. For instance, elastic instabilities coupled with the discrete character of the interface are responsible for the dramatic sinking of granular rafts [16,30,31], implying that large deformations of such rafts lead to different behaviors. Thus, the intrinsic discrete nature of such objects is a key parameter and it is crucial to question the validity and test the limitations of the analogy of such composite materials with elastic sheets.

In this Rapid Communication, we first present an experiment where a wrinkle-to-fold transition is observed in particle monolayers at a liquid-fluid interface, suggesting that such systems behave as an elastic sheet. However, by investigating further the limits of a continuum elastic model, we reveal that these composite materials also have very specific mechanical properties: For large deformations, what seems to be at first a pure elastic response is in fact an irreversible plastic transition that can only be rejuvenated through an annealing stirring process.

Rafts are made by carefully sprinkling dense particles above a planar liquid-fluid interface. The particles straddle either an oil-water or air-water interface where they are trapped and aggregate, forming a monolayer of particles, the granular raft. Most of the experiments were conducted with polydisperse beads in zirconium oxide, $\mathrm{ZrO}\left(\rho_{s}=3.8 \mathrm{~g} \mathrm{~cm}^{-3}\right)$, from Glen Mills, Inc., or coated glass, $\mathrm{SiO}\left(\rho_{s}=2.5 \mathrm{~g} \mathrm{~cm}^{-3}\right)$, from Sigmund Lindner. The water is de-ionized, the oil is light mineral oil (Sigma Aldrich) of density $\rho_{o}=0.838 \mathrm{~g} \mathrm{~cm}^{-3}$, and the interfacial tension is $\gamma_{o / w}=46 \mathrm{mN} / \mathrm{m}$. The particle diameters and oil-water contact angles $\theta_{Y}$ vary in the range $20<d(\mu \mathrm{m})<875$ and $80<\cos \theta_{Y}(\mathrm{deg})<160$ (see Supplemental Material [32]). A typical freely floating granular raft consists of a flat central region below the water surface and curved menisci at its edges. Its stability and shape have been studied in detail [16] and are determined by a balance between gravity, buoyancy effects, and surface tension. In particular, the depth of the flat region is determined by the buoyancy and weight of the particles at equilibrium.

To compress the raft, a glass plate is mounted to a step motor of micrometer precision (Thorlabs) that moves uniaxially, enabling its incremental compression along the $x$ axis [Fig. 1(a)]. Compression is controlled in steps of $200 \mu \mathrm{m}$ every $10 \mathrm{~s}$ to let the raft relax to its equilibrium shape at each step. The raft is imaged from the side and the bottom with two cameras (Nikon D800E), and fringes are projected on its surface to reconstruct it using Fourier transform profilometry [32-34]. At first, when the raft is compressed, the particles have enough space to rearrange and the raft elongates along the $z$ axis in order to accommodate the compression. Then, when the particles are confined along the interface, grain-to-grain interactions make rearrangements difficult and the raft starts to buckle out of plane: We observe pseudosinusoidal deformations of wavelength $\lambda$ along the raft which are perpendicular to the direction of compression 


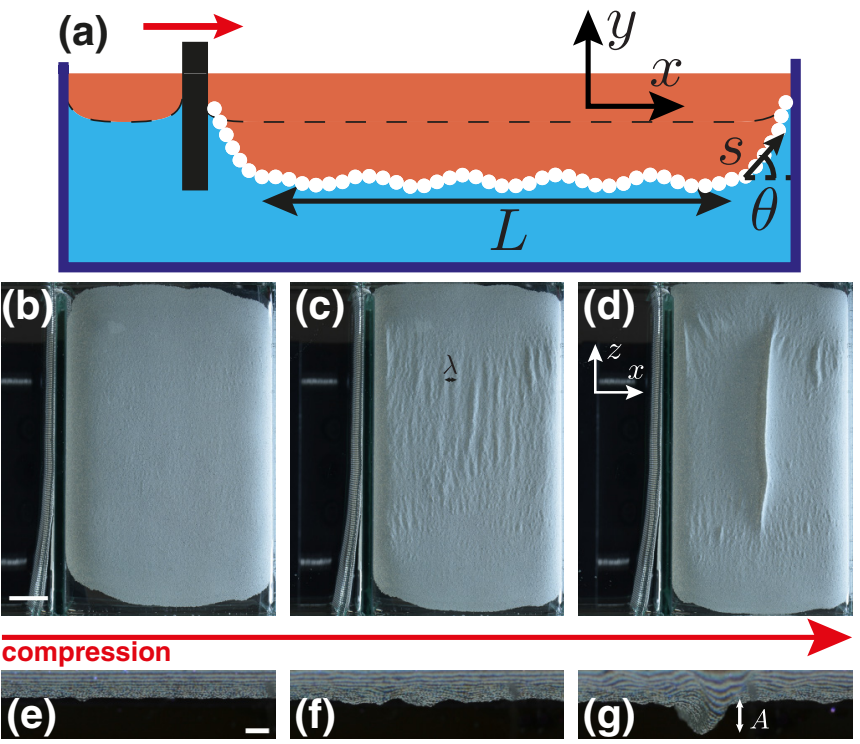

FIG. 1. (a) Schematic of experiment. The dashed line represents the water surface in the absence of a raft. The compression $\Delta$ is determined with $L$, the length of the flat part of the raft (without the menisci). Bottom pictures [(b)-(d), scale bar $1 \mathrm{~cm}$ ] and side pictures [(e)- $(\mathrm{g})$, scale bar $1 \mathrm{~mm}]$ of two granular rafts showing small wrinkles, large wrinkles, and the fold. Compression increases from left to right, $d=150 \mu \mathrm{m}$. (See the video [32].)

[Figs. 1(c) and 1(f)] until finally at a critical compression these small deformations localize into one large fold [Figs. 1(d) and $1(\mathrm{~g})]$ (see the full movie [32]).

To quantify this fold formation we measure the compression imposed to the raft. When the raft reaches the solid boundaries, particles climb up the menisci whose shape is defined by the wall's wetting properties. It thus forms a complex structure depending on both the wall and raft characteristics which can rotate and bend during the compression, absorbing and releasing large stresses and strains (see Fig. 2 and details in Ref. [32]). To simplify the problem, we only consider the large flat region (of initial aspect ratio $\sim 0.5-0.7$ ) that is
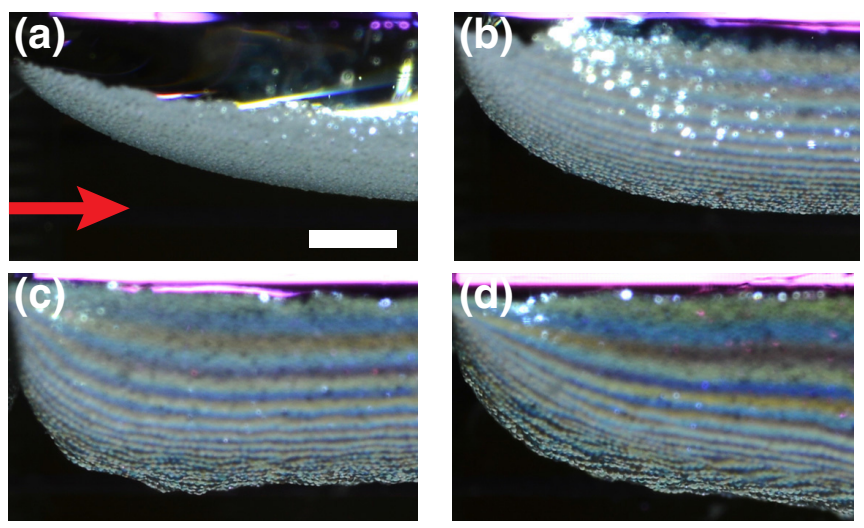

FIG. 2. Closeup view of a raft meniscus during a typical experiment $(\mathrm{ZrO}, d=150 \mu \mathrm{m})$; the compressing wall is located at the left edge of each image and the arrow indicates the direction of compression. Compression increases from (a) to (d); scale bar: $2 \mathrm{~mm}$.

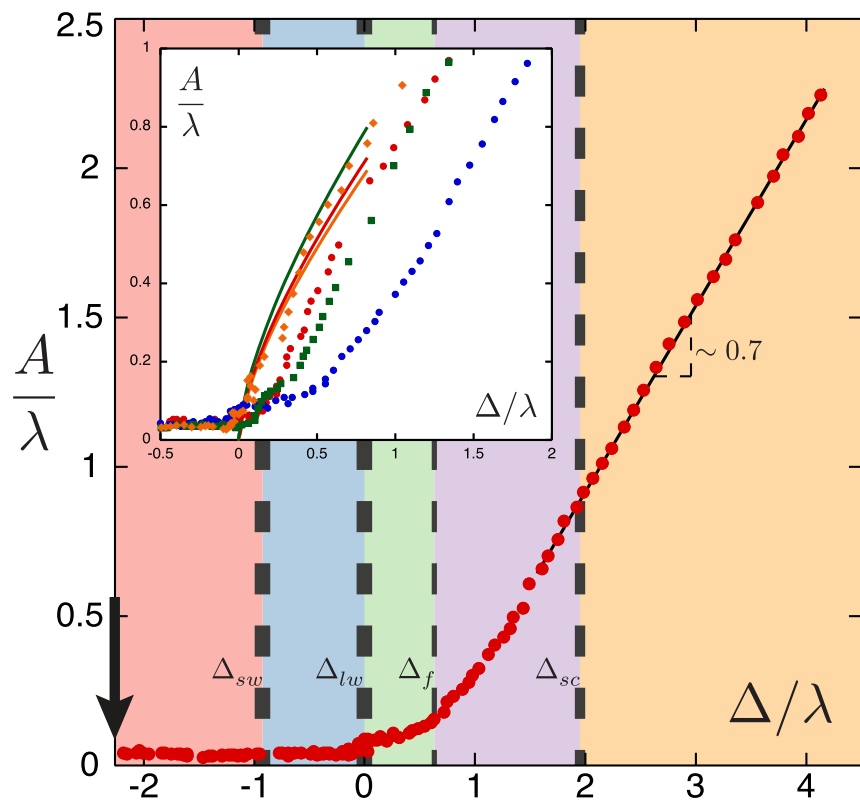

FIG. 3. Dimensionless amplitude as a function of the dimensionless compression. The five distinct phases are delimited, with the thickness of the dashed delimiting line indicating the uncertainty on the transition compressions. Inset: Dimensionless amplitude as a function of the dimensionless compression for four different experiments. Red and blue circles are for $\mathrm{ZrO}, d=150 \mu \mathrm{m}$, green squares are for $\mathrm{ZrO}, d=250 \mu \mathrm{m}$, and orange diamonds are for $\mathrm{SiO}$, $d=500 \mu \mathrm{m}$. The three solid lines are the result of Eq. (1), with the values of $M$ corresponding to the data (same color): $M=3.25,5.01$, 2.65 .

always present in the center [Fig. 1(a)]. By analogy with the compression of an elastic sheet, we define the confinement as $\Delta=L_{0}-L, L$ corresponding to the length of that flat region and $L_{0}$ its length when the wrinkles in Fig. 3(c) are first observed. We further measure the amplitude of the wrinkles $A$ as we increase the confinement incrementally (see Fig. 3 for a typical experiment with $\mathrm{ZrO}$ particles at an oil-water interface, $d=150 \mu \mathrm{m}$ ). Five different regions are identified: In region I (the raft edges touch both plates; black arrow in Fig. 3) the particles rearrange as explained above. At a critical $\Delta_{s w} / \lambda=0.92$ starts region II where a careful examination reveals small undulations in some regions of the raft surface [of wavelength $\lambda_{s}<\lambda$; Fig. 4(a)]. These small wrinkles have a small lateral extension (along $z$ ) and their amplitude is smaller than the resolution of our measurement (roughly $d / 2$ ). They gradually appear on the whole raft as we increase compression until $\Delta_{l w} / \lambda=0$. Above this threshold (region III), the wrinkles, of wavelength $\lambda$, grow in length and amplitude [Figs. 1(c) and 1(f)]. Then an abrupt transition occurs around $\Delta_{f} / \lambda \approx 0.6$ : One of the wrinkles starts to grow much faster than the others, thus creating a large fold at the center of the raft [Figs. $1(\mathrm{~d})$ and $1(\mathrm{~g})$, region IV]. This fold grows in amplitude and along the $z$ direction upon further compression while the other wrinkles disappear progressively (see Ref. [32]). Its amplitude then grows linearly with compression, indicating that all of the deformation is now localized in the fold (Fig. 3). At $\Delta_{s c} / \lambda \approx 2.0$ both sides of the fold come into contact, encapsulating a small oil volume 

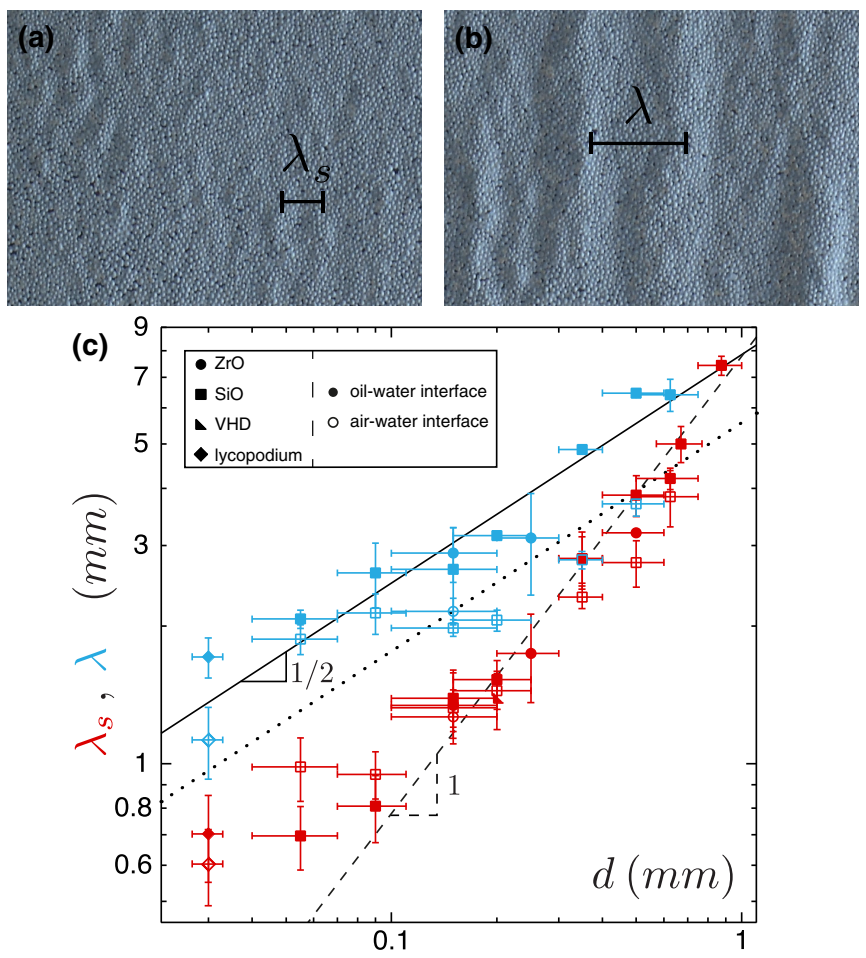

FIG. 4. Pictures of (a) small and (b) large wrinkles for a $\mathrm{ZrO}$ raft, $d=150 \mu \mathrm{m}$. Here, $\lambda_{s} \approx 1.3 \mathrm{~mm}$ and $\lambda \approx 2.9 \mathrm{~mm}$. (c) Small wrinkle $\left(\lambda_{s}\right.$, red symbols) and large wrinkle ( $\lambda$, blue symbols) wavelengths as a function of the particle size. The symbols indicates the particle material with different densities $\left(\rho_{\mathrm{VHD}}=6.0 \mathrm{~g} \mathrm{~cm}^{-3}\right.$ and $\rho_{\text {lyc }}=1.2 \mathrm{~g} \mathrm{~cm}^{-3}$ ) and contact angle. Solid symbols are for oil-water experiments and open ones for air-water experiments. The large wavelength is fitted to $\lambda=3.39 \sqrt{\ell_{c} d}$. The solid line is the fit for oil-water experiments $\left(\ell_{c}=5.4 \mathrm{~mm}\right)$ while the dotted line is the fit for air-water experiments $\left(\ell_{c}=2.7 \mathrm{~mm}\right)$. The dashed line is a guide to the eye.

(region V). Finally, the last point of the curve corresponds to the critical compression at which most of the raft's weight is pulled into the fold, leading to the raft destabilization.

By varying liquid and particle properties, we observe that $\lambda_{s}$ only varies linearly with the particle diameter $d$ [Fig. 4(c)]. This is similar to what is observed for a particle monolayer stuck to an elastic solid [35], revealing the discrete nature of the granular raft [36]. By contrast, when measuring the large wrinkles' wavelength, $\lambda$ varies with $d^{1 / 2}$ and depends on the liquids used, but not on the particle density or on the contact angle. These types of wrinkles have an elastic origin and have already been observed on compressed particle rafts [15,37]. The elastic description of the interface [15] indeed predicts $\lambda=$ $4.84 \sqrt{\ell_{c} d}$ (with $\ell_{c}=\sqrt{\gamma / \rho g}$ the capillary length) while our data are well fitted by $\lambda=3.39 \sqrt{\ell_{c} d}$ [Fig. 4(c)], confirming the dependence on $d$ and also showing the variation with $\ell_{c}$.

Keeping the elastic analogy for the particle raft, we describe it as a continuous heavy elastic sheet of length $L_{0}$, width $W$, thickness $d$, density $\rho_{\text {eff }}$, and bending rigidity $B$ (per unit width) floating between two liquids (of density $\rho_{w}$ and $\rho_{o}$ ) [38]. This approach generalizes the simple first model that was derived for axisymmetric membranes where only isotropic tension was considered, that could not capture the formation of wrinkles and thus the elastic nature of the rafts [16]. More precisely, it consists of adding the grains' weight to the model developed for a two-dimensional (2D) Euler-Bernoulli beam floating between two liquids [1,39] and assume thus the invariance of the raft in the width direction. The elastic feature of the granular raft has different origins that act as additional contributions: The first one comes from the interface menisci between the grains whose deformation generates elasticity (with an associated Young modulus $E \sim \gamma_{o / w} / d$ ) [15]; in addition, the chain forces that are present in granular systems are also known to lead to elastoplastic behavior of the raft $[27,40]$. The balance of dimensionless internal forces $\left(n_{x}, n_{y}\right)$ and bending moment $m$, along with the kinematic and bending constitutive relation, yield

$$
\begin{aligned}
\partial_{s} x & =\cos \theta, \quad \partial_{s} n_{x}=-y \sin \theta, \\
\partial_{s} y & =\sin \theta, \quad \partial_{s} n_{y}=y \cos \theta+M, \\
m & =\partial_{s} \theta, \quad \partial_{s} m=n_{x} \sin \theta-n_{y} \cos \theta .
\end{aligned}
$$

Here, the intrinsic coordinates $(s, \theta)$ are the arclength and the local angle between the raft and the horizontal axis, respectively [see Fig. 1(a)]. The sheet centerline is parametrized by $[x(s), y(s)]$. Here, the surface tension is embedded in the internal forces $\mathbf{n}$ acting in the raft, that account also for the contact force between the grains. The system has been made dimensionless by dividing all lengths by $\ell_{e h}=\left(\frac{B}{\left(\rho_{w}-\rho_{o}\right) g}\right)^{1 / 4}=$ $\frac{\lambda}{2 \pi}$, forces by $\frac{B W}{\ell_{e h}^{2}}$, and moments by $\frac{B W}{\ell_{e h}}$. Since we neglect the deformation of the raft menisci in the experiments, we also remove them from the model in order to solve the equation only for the flat portion of the sheet located below the water surface. The dimensionless boundary conditions read

$$
\begin{array}{ll}
y(0)=-M, & y\left(L_{0}\right)=-M, \\
\theta(0)=0, & \theta\left(L_{0}\right)=0, \\
x(0)=0, & x\left(L_{0}\right)=L_{0}-\Delta,
\end{array}
$$

where $M=\frac{\rho_{\text {eff }}}{\rho_{w}-\rho_{o}} \frac{d}{\ell_{e h}}$ is the dimensionless parameter introduced in Ref. [38] that compares the weight of the sheet to the restoring force provided by the fluids displaced over the length $\ell_{e h}$. The boundary condition at the edges of the sheet $y(0)=y\left(L_{0}\right)=-M$ simply means that the sheet is clamped at its freely floating location, i.e., where its weight is balanced by the displaced fluid. We solve numerically the system of equations (1) with the boundary conditions (2) using the MATLAB routine BVP5C with a continuation algorithm. The raft bending rigidity is determined using the experimental $\lambda$ through $B=\left(\rho_{w}-\rho_{o}\right) g\left(\frac{\lambda}{2 \pi}\right)^{4}$. The effective density takes into account the voids in the sheet and the fact that the particles are immersed. For a monolayer of spherical monodisperse particles half immersed in oil and water $\rho_{\text {eff }}=\frac{2}{3} \phi\left(\rho_{s}-\frac{\rho_{o}+\rho_{w}}{2}\right)$, where $\phi$ is the $2 \mathrm{D}$ packing fraction $(\phi \approx 0.84$ for jammed polydisperse systems, and the $2 / 3$ factor accounts for the $3 \mathrm{D}$ volume corresponding to this $2 \mathrm{D}$ packing fraction). To compare the data with the theoretical model, we plot the dimensionless amplitude as a function of compression at the wrinkle-to-fold transition for different types of particles (see the inset in Fig. 3). At first glance, the variation of the fold amplitude during compression is well captured by the continuous description, with no adjustable 

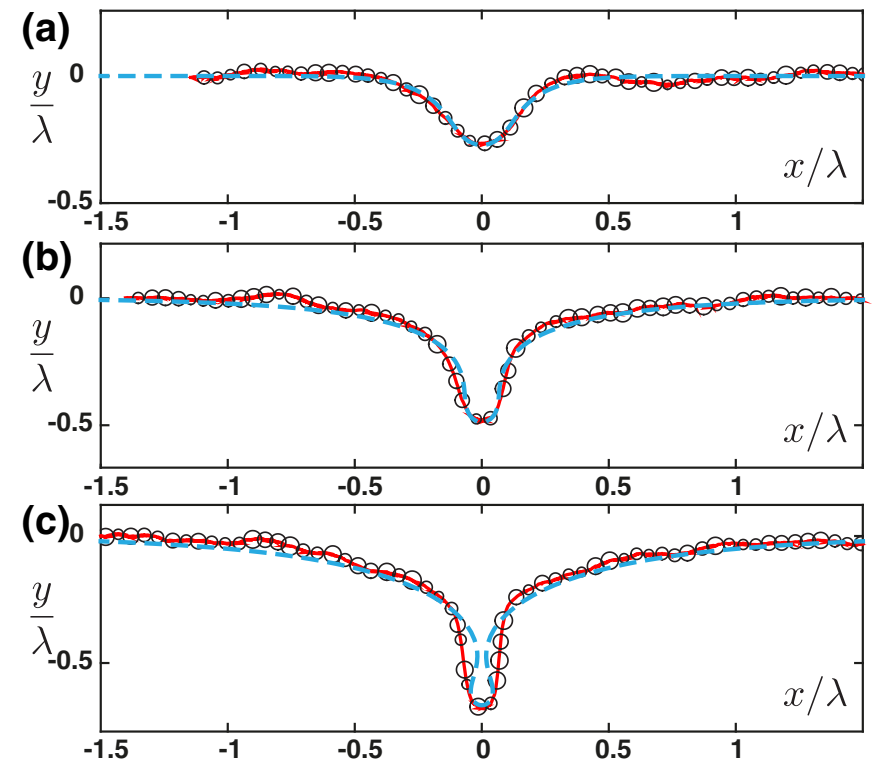

FIG. 5. Comparison of the experimental raft profile measured with Fourier transform profilometry (red solid curve) and the solution of Eq. (1) (blue dashed curve) at the same amplitude (different compression) for $M=3.25$. The typical particle size is drawn $(d=150 \pm 50 \mu \mathrm{m})$ as black open circles. (a) $(\Delta / \lambda)_{\text {expt }}=$ $0.42,(\Delta / \lambda)_{\text {num }}=0.18$. (b) $(\Delta / \lambda)_{\text {expt }}=0.64,(\Delta / \lambda)_{\text {num }}=0.44$. (c) $(\Delta / \lambda)_{\text {expt }}=0.92,(\Delta / \lambda)_{\text {num }}=0.72$.

parameter. However, while this elastic continuous sheet model predicts that the wrinkle-to-fold transition always occurs around $\Delta_{f} / \lambda \approx 0.03$ [32], we find experimentally higher values. In addition, two identical rafts may buckle at a different $\Delta_{f}$. Such behavior is a signature of the granular nature of this composite material, where individual particles rearrange during the compression process, leading to inhomogeneous stress and strain repartition, inducing jamming, frustration, and residual chain forces in the system [22,27,40]. This can be clearly seen in Fig. 1(d) where the fold is already formed while some wrinkles are still present. Interestingly, when we compare the experimental and theoretical (using the continuous elastic model) fold profiles at the same amplitude $A$ (and thus not formally obtained for the same global compression $\Delta$ ), we observe a good quantitative agreement, as shown in Figs. 5(a)-5(c). In particular, the agreement between the two profiles is good in region IV [Figs. 5(a) and 5(b)], validating that the compression difference is due to rearrangements that do not affect the fold formation. As we reach self-contact (region $\mathrm{V}$ ), the fold in the model forms a loop not observed experimentally [see Fig. 5(c)]. Because the model represents the sheet centerline and does not account its thickness $d$, self-contact occurs when the neck width is equal to $d$ and stops the loop formation. Since $d$ is roughly the size of the final loop and the polydispersity of our particles is important, we observe a vertical fold of width $\sim 3 d$ after self-contact. The model cannot describe the full raft profile beyond the critical compression $\Delta_{s c}$ [end of black curves in the inset in Fig. 3(a)] since the numerical fold then starts to interpenetrate. However, in region $\mathrm{V}$, the fold profiles can still be described outside of the self-contact zone using the elastic sheet model only by changing the boundary conditions

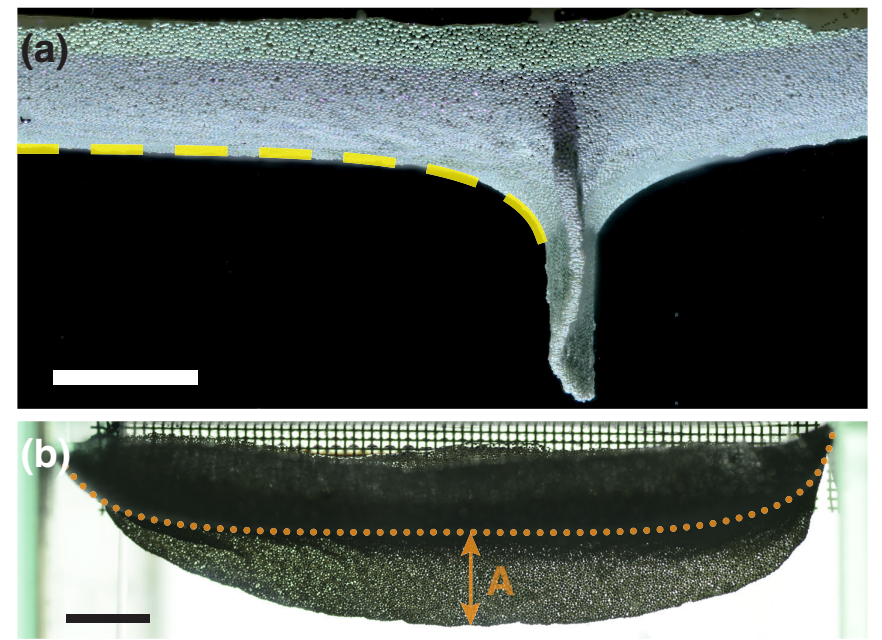

FIG. 6. Post-self-contact fold ( $\mathrm{ZrO}, d=150 \mu \mathrm{m})$; both scale bars are $5 \mathrm{~mm}$. (a) Side view perpendicular to the direction of compression (along $z$ ). The portion of the raft not in self-contact evolves as $\Delta$ increases but keeps a self-similar shape, which is also well reproduced by the model [Eq. (1) with modified boundary conditions, yellow dashed curve; see Ref. [32]]. (b) View in the direction of compression (along $x$ ). The dotted line shows the undeformed flat raft profile, far from the fold.

appropriately, exhibiting a self-similar evolution with $\Delta-\Delta_{s c}$ [dashed line of Fig. 6(a) [32]]. Figure 6(b) presents the lateral structure of the fold, showing that the fold is also localized in this direction, as it could be observed in Fig. 1(d). This shape is due to stress inhomogeneities, the edge of the fold here being less confined. At the center of the fold, where we extract the profiles and measure $A$, the raft's depth only varies slowly.

To investigate the influence of particle rearrangements, we study the reversibility of the folding process by performing cycles of compression and unloading of the raft, being careful not to reach the critical compression at which the raft destabilizes. When we plot the evolution of the amplitude during each cycle we observe a hysteresis behavior (Fig. 7). The first compression is similar to the one described earlier [Fig. 7(1)]. Then, as the confinement is decreased progressively, the raft unfolds and recovers a flat surface without going through the wrinkled state. In addition, a small "scar" remains present at the initial fold position even when the compression is completely released, as it can be seen in Fig. 7(2): There, the particles seem to be aligned along the scar. During the second compression cycle the raft localizes directly into a single fold exactly at the scar location [Fig. 7(3)], without going through a wrinkle-to-fold transition. Furthermore, if compression and relaxation cycles are repeated further on, they always form the same fold and follow after few cycles the same curves (close to the curves 2 and 3 in the diagram of Fig. 7). This behavior suggests that the granular raft exhibits a plastic irreversible transition when the fold reaches the self-contact that acts as the plastic threshold. The fold can be considered here as an analog to a ridge in crumpled papers [41]. It is tempting therefore to associate this plastic transition to the elastoplastic behavior of grains under compaction that exhibit a network of intense force chains $[27,40]$. However, if we stir thoroughly the raft to force a new random particle arrangement, we recover the behavior 

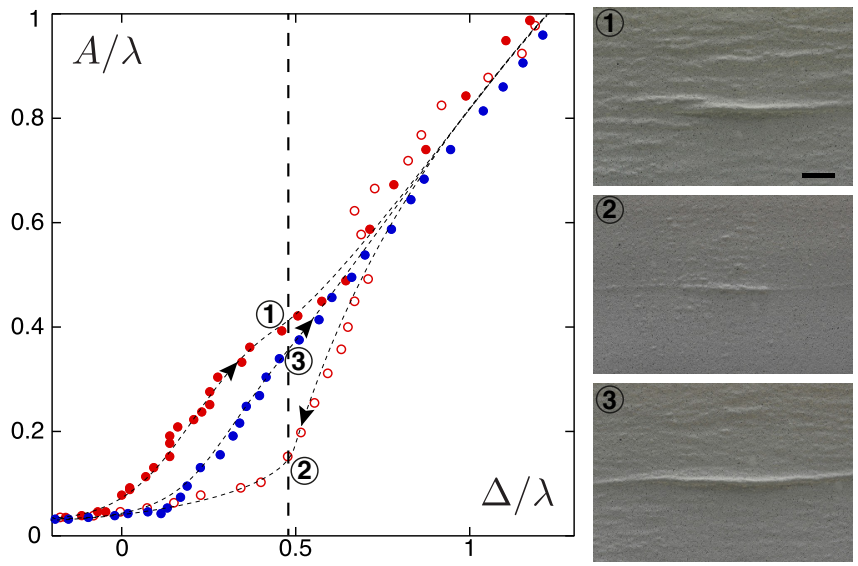

FIG. 7. Dimensionless amplitude as a function of the dimensionless compression for loading cycles on a $\mathrm{ZrO}$ raft $(d=150 \mu \mathrm{m})$ at the oil-water interface. The dashed lines and arrows are guides to the eye. (1)-(3) Corresponding bottom pictures taken at the same compression $\Delta / \lambda=0.47$ for different compression cycles: (1) first loading, (2) first unloading, and (3) second loading. Scale bar: $5 \mathrm{~mm}$. observed when the raft is first compressed: The raft elastic property is recovered through this annealing process similarly to what is observed in amorphous materials [42], spin glasses [43], vibrated grains [44], or shape memory polymers [45]. In our system, the annealing process is probably related to the breakdown of the chain forces that are related to the jamming of the granular raft [23-25].

In conclusion, we show in this Rapid Communication that under large compression a granular raft deviates from the elastic sheet model since it undergoes an irreversible plastic transition. This transition is different from the reversible wrinkle-to-fold one observed also for elastic sheets. Moreover, the particle rearrangements act as an effective temperature in the system that can anneal the plastic transition. This composite material, which is made of interacting grains at the interface, share the properties of elastoplastic sheets, as well as amorphous and discrete materials.

The authors thank Manouk Abkarian for many valuable discussions at the early stages of this work and Sébastien Neukirch for fruitful conversations concerning the model.
[1] L. Pocivavsek, R. Dellsy, A. Kern, S. Johnson, B. Lin, K. Y. C. Lee, and E. Cerda, Stress and fold localization in thin elastic membranes Science 320, 912 (2008).

[2] B. D. Leahy, L. Pocivavsek, M. Meron, K. L. Lam, D. Salas, P. J. Viccaro, K. Y. C. Lee, and B. Lin, Geometric Stability and Elastic Response of a Supported Nanoparticle Film, Phys. Rev. Lett. 105, 058301 (2010).

[3] F. Brau, H. Vandeparre, A. Sabbah, C. Poulard, A. Bouadoud, and P. Damman, Multiple-length-scale elastic instability mimics parametric resonance of nonlinear oscillators, Nat. Phys. 7, 56 (2011).

[4] P. Kim, M. Abkarian, and H. A. Stone, Hierarchical folding of elastic membranes under biaxial compressive stress, Nat. Mater. 10, 952 (2011).

[5] M. Piñeirua, N. Tanaka, B. Roman, and J. Bico, Capillary buckling of a floating annulus, Soft Matter 9, 10985 (2013).

[6] J. D. Paulsen, V. Démery, K. B. Toga, Z. Qiu, T. P. Russell, B. Davidovitch, and N. Menon, Geometry-Driven Folding of a Floating Annular Sheet, Phys. Rev. Lett. 118, 048004 (2017).

[7] E. Cerda and L. Mahadevan, Geometry and Physics of Wrinkling, Phys. Rev. Lett. 90, 074302 (2003).

[8] D. D. Pollard and R. C. Fletcher, Fundamentals of Structural Geology (Cambridge University Press, Cambridge, U.K., 2005).

[9] T. Tallinen, J. Y. Chung, F. Rousseau, N. Girard, J. Lefevre, and L. Mahadevan, On the growth and form of cortical convolutions, Nat. Phys. 12, 588 (2016).

[10] T. Boatwright, A. J. Levine, and M. Dennin, Tracking giant folds in a monolayer, Langmuir 26, 12755 (2010).

[11] M. Trejo, C. Douarche, V. Bailleux, C. Poulard, S. Mariot, C. Regeard, and E. Raspaud, Elasticity and wrinkled morphology of bacillus subtilis pellicles, Proc. Natl. Acad. Sci. USA 110, 2011 (2013).

[12] D. G. Schultz, X.-M. Lin, D. Li, J. Gebhardt, M. Meron, J. Viccaro, and B. Lin, Structure, wrinkling, and reversibility of langmuir monolayers of gold nanoparticles, J. Phys. Chem. B 110, 24522 (2006).

[13] K. E. Mueggenburg, X.-M. Lin, R. H. Goldsmith, and H. M. Jaeger, Elastic membranes of close-packed nanoparticle arrays, Nat. Mater. 6, 656 (2007).

[14] B. P. Binks, Particles as surfactants-similarities and differences, Curr. Opin. Colloid Interface Sci. 7, 21 (2002).

[15] D. Vella, P. Aussillous, and L. Mahadevan, Elasticity of an interfacial particle raft, Europhys. Lett. 68, 212 (2004).

[16] S. Protière, C. Josserand, J. M. Aristoff, H. A. Stone, and M. Abkarian, Sinking a Granular Raft, Phys. Rev. Lett. 118, 108001 (2017).

[17] P. Cicuta, E. J. Stancik, and G. G. Fuller, Shearing or Compressing a Soft Glass in 2D: Time-Concentration Superposition, Phys. Rev. Lett. 90, 236101 (2003).

[18] C. Monteux, J. Kirkwood, H. Xu, E. Jung, and G. G. Fuller, Determining the mechanical response of particle-laden fluid interfaces using surface pressure isotherms and bulk pressure measurements of droplets, Phys. Chem. Chem. Phys. 9, 6344 (2007).

[19] C. Planchette, E. Lorenceau, and A.-L. Biance, Surface wave on a particle raft, Soft Matter 8, 2444 (2012).

[20] A. Varshney, A. Sane, S. Ghosh, and S. Bhattacharya, Amorphous to amorphous transition in particle rafts, Phys. Rev. E 86, 031402 (2012).

[21] G. Lagubeau, A. Rescaglio, and F. Melo, Armoring a droplet: Soft jamming of a dense granular interface, Phys. Rev. E 90 , 030201 (2014).

[22] A. J. Liu and S. R. Nagel, Nonlinear dynamics: Jamming is not just cool any more, Nature (London) 396, 21 (1998).

[23] C. S. O’Hern, L. E. Silbert, A. J. Liu, and S. R. Nagel, Jamming at zero temperature and zero applied stress: The epitome of disorder, Phys. Rev. E 68, 011306 (2005). 
[24] A. J. Liu and S. R. Nagel, The jamming transition and the marginally jammed solid, Annu. Rev. Condens. Matter Phys. 1, 347 (2010).

[25] D. Bi, J. Zhang, B. Chakraborty, and R. P. Behringer, Jamming by shear, Nature (London) 480, 355 (2011).

[26] F. Radjai, M. Jean, J.-J. Moreau, and S. Roux, Force Distributions in Dense Two-Dimensional Granular Systems, Phys. Rev. Lett. 77, 274 (1996).

[27] M. E. Cates, J. P. Wittmer, J.-P. Bouchaud, and P. Claudin, Jamming, Force Chains, and Fragile Matter, Phys. Rev. Lett. 81, 1841 (1998).

[28] A. B. Subramaniam, M. Abkarian, and H. A. Stone, Controlled assembly of jammed colloidal shells on fluid droplets, Nat. Mater. 4, 553 (2005).

[29] P. Cicuta and D. Vella, Granular Character of Particle Rafts, Phys. Rev. Lett. 102, 138302 (2009).

[30] M. Abkarian, S. Protière, J. M. Aristoff, and H. A. Stone, Gravity-induced encapsulation of liquids by destabilization of granular rafts, Nat. Commun. 4, 1895 (2013).

[31] D. Vella, Floating versus sinking, Annu. Rev. Fluid Mech. 47, 115 (2015).

[32] See Supplemental Material at http://link.aps.org/supplemental/ 10.1103/PhysRevMaterials.1.042601 for materials and methods, more details on the wrinkle-to-fold transition and the self-similar profile post-self-contact, as well as a movie of the experiment.

[33] M. Takeda and K. Mutoh, Fourier transform profilometry for the automatic measurement of 3-D object shapes, Appl. Opt. 22, 3977 (1983).

[34] P. J. Cobelli, A. Maurel, V. Pagneux, and P. Petitjeans, Global measurement of water waves by Fourier transform profilometry, Exp. Fluids 46, 1037 (2009).
[35] A. Tordesillas, D. Carey, A. B. Croll, J. Shi, and B. Gurmessa, Micromechanics of elastic buckling of a colloidal polymer layer on a soft substrate: Experiment and theory, Granular Matter 16, 249 (2014).

[36] N. Taccoen, F. Lequeux, D. Z. Gunes, and C. N. Baroud, Probing the Mechanical Strength of an Armored Bubble and its Implication to Particle-Stabilized Foams, Phys. Rev. X 6, 011010 (2016).

[37] T. D. Kassuga and J. P. Rothstein, Buckling of particle-laden interfaces, J. Colloid Interface Sci. 448, 287 (2015).

[38] E. Jambon-Puillet, D. Vella, and S. Protiere, The compression of a heavy floating elastic film, Soft Matter 12, 9289 (2016).

[39] M. Rivetti and S. Neukirch, The mode branching route to localization of the finite-length floating elastica, J. Mech. Phys. Solids 69, 143 (2014).

[40] J.-P. Bouchaud, P. Claudin, D. Levine, and M. Otto, Force chain splitting in granular materials: A mechanism for large-scale pseudo-elastic behaviour, Eur. Phys. J. E. 4, 451 (2001).

[41] S. Deboeuf, E. Katzav, A. Boudaoud, D. Bonn, and M. AddaBedia, Comparative Study of Crumpling and Folding of Thin Sheets, Phys. Rev. Lett. 110, 104301 (2013).

[42] J.-Y. Raty, W. Zhang, J. Luckas, C. Chen, R. Mazzarello, C. Bichara, and M. Wuttig, Aging mechanisms in amorphous phase-change materials, Nat. Commun. 6, 7467 (2015).

[43] F. Alberici-Kious, J.-P. Bouchaud, L.F. Cugliandolo, P. Doussineau, and A. Levelut, Aging in $\mathrm{K}_{1-x} \mathrm{Li}_{x} \mathrm{TaO}_{3}$ : A Domain Growth Interpretation, Phys. Rev. Lett. 81, 4987 (1998).

[44] C. Josserand, A. Tkachenko, D. Mueth, and H. Jaeger, Memory Effects in Granular Materials, Phys. Rev. Lett. 85, 3632 (2000).

[45] F. Pilate, A. Toncheva, P. Dubois, and J.-M. Raquez, Shapememory polymers for multiple applications in the materials world, Eur. Polym. J. 80, 268 (2016). 